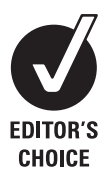

See Editorial, p 4

International Centre for Circulatory Health, Imperial College London and St Mary's Hospital, London, UK

Correspondence to: Dr C E Raphael, International Centre for Circulatory Health, St Mary's Hospital and Imperial College, 59 North Wharf Rd, London, UK; claire.raphael@ gmail.com

Accepted 13 May 2008 Published Online First 15 August 2008

\title{
Quantifying the paradoxical effect of higher systolic blood pressure on mortality in chronic heart failure
}

\author{
C E Raphael, Z I Whinnett, J E Davies, M Fontana, E A Ferenczi, C H Manisty, J Mayet, \\ D P Francis
}

\begin{abstract}
Background: Although higher blood pressures are generally recognised to be an adverse prognostic marker in risk assessment of cardiology patients, its relationship to risk in chronic heart failure (CHF) may be different.
\end{abstract}

Objective: To examine systematically published reports on the relationship between blood pressure and mortality in $\mathrm{CHF}$.

Methods: Medline and Embase were used to identify studies that gave a hazard or relative risk ratio for systolic blood pressure in a stable population with CHF. Included studies were analysed to obtain a unified hazard ratio and quantify the degree of confidence.

Results: 10 studies met the inclusion criteria, giving a total population of 8088, with 29222 person-years of follow-up. All studies showed that a higher systolic blood pressure (SBP) was a favourable prognostic marker in $\mathrm{CHF}$, in contrast to the general population where it is an indicator of poorer prognosis. The decrease in mortality rates associated with a $10 \mathrm{~mm} \mathrm{Hg}$ higher SBP was $13.0 \%$ (95\% Cl $10.6 \%$ to $15.4 \%$ ) in the heart failure population. This was not related to aetiology, ACE inhibitor or $\beta$ blocker use.

Conclusion: SBP is an easily measured, continuous variable that has a remarkably consistent relationship with mortality within the CHF population. The potential of this simple variable in outpatient assessment of patients with CHF should not be neglected. One possible application of this information is in the optimisation of cardiac resynchronisation devices.

High mortality and poor quality of life continue to characterise a diagnosis of heart failure despite maximal pharmacological treatment. ${ }^{1}$ The number of patients with heart failure will continue to rise as the population ages and survival from myocardial infarction continues to improve.

Effective measures of prognosis are required to aid doctors with the timing of introduction of treatments and may also be useful as a measure of response to these treatments. Echocardiography and cardiopulmonary exercise testing have been shown to provide a useful measure of prognosis in this condition. However, the availability of these tests is still limited and repeated testing for individual patients is often restricted by time and cost constraints.

Systolic blood pressure (SBP) is by contrast, a simple bedside test which is recorded routinely at most cardiology appointments, can be tracked longitudinally over time and requires minimal equipment and training to assess. It therefore has the potential to be a simple method of assessing prognosis to enable doctors to target treatment and potentially assess the impact therapies.

While it is well recognised that high SBP is an adverse prognostic marker in risk assessment of most cardiac patients, ${ }^{2}{ }^{3}$ its relationship with mortality in the population with chronic heart failure (CHF) is less clear. Management of heart failure includes use of angiotensin converting enzyme inhibitors (ACEi) and $\beta$ blockers, both of which have been shown to improve prognosis in heart failure but which also lower the blood pressure. A large cohort study in patients admitted to hospital with acute heart failure showed that lower blood pressure is associated with a higher mortality. ${ }^{4}$

However, within the population with chronic heart failure, little prospective information has been collected to examine the relationship between SBP and mortality. A retrospective cohort study showed that a lower SBP was associated with higher mortality, although it suggested that there is a non-linear, possibly J-shaped relationship between SBP and mortality. ${ }^{5}$ Several small studies, but no large-scale studies, have looked at the relationship between mortality and SBP.

Blood pressure is intrinsically related to the heart's ability to pump, and the mean arterial blood pressure is proportional to the cardiac output. Therefore, physiologically, there is a strong case for blood pressure as a marker of prognosis in heart failure.

This study therefore aimed to examine and quantify the relationship between SBP and mortality for stable patients with $\mathrm{CHF}$.

\section{METHODS}

A literature search was performed using the key words "heart failure" and "prognosis" or "mortality" using the Embase and PubMed databases. These search terms were chosen to minimise bias so that studies that did not focus on blood pressure as the primary outcome measure and studies in which there was a non-significant relationship between blood pressure and mortality would be included.

The inclusion criteria were studies performed in a population with CHF with patients who were medically stable for at least 1 month before entry into the trial and had appropriate investigations confirming their diagnosis. Patients had to have had their SBP measured at the start of the trial.

Studies that provided sufficient statistical data to calculate the univariate mortality hazard ratio for a known unit change in SBP were included and authors for studies that did not include sufficient 
information were contacted to see if further information was available. Two reviewers assessed each of the papers identified. There were no disagreements between the reviewers over selection of papers or extraction of data.

\section{Statistical analysis}

The univariate hazard ratios for mortality and 95\% confidence intervals were extracted from each of the included studies. Since different papers quoted mortality hazard ratios for different units of SBP, such as 1 standard deviation, the results were standardised to a 10-unit increase in SBP. To do this, the formula

$$
H R_{10 m m ~ H g}=\left(H R_{x m m ~ H g}\right)^{\left(\frac{10}{x}\right)}
$$

was applied where $\mathrm{HR}_{\mathrm{x}}$ is the hazard ratio quoted in the source document and $\mathrm{x}$ is the increase in SBP in $\mathrm{mm} \mathrm{Hg}$. So, for example, for a 5-unit increase in SBP with a hazard ratio of 0.91 , $\mathrm{x}=5$ and $\mathrm{HR}_{\mathrm{x}}=0.91$, giving a standardised hazard ratio of 0.83 per 10-unit increase in SBP. The confidence intervals for each hazard ratio were recalculated in the same way.

A fixed effects statistical analysis model was used to pool the data. Each study was assigned a relative weight, calculated from the reciprocal of the square of the width of the confidence interval, where the confidence interval was expressed in logarithmic units for a 10-unit increase in SBP. The hazard ratios for each study were then expressed in log units, and the weighted mean was calculated from the sum of the log of the hazard ratio per unit SBP multiplied by the weighting for each study. The logarithmic width of the confidence interval for the weighted mean was $1 / \sqrt{ }($ Lweight $)$.

Two studies (Huynh ${ }^{14}$ and Davos ${ }^{12}$ ) met the inclusion criteria but the lower limit of the hazard ratio for mortality had been rounded so that it was equal to the point estimate for the hazard ratio-for example, 0.99 (0.99 to 1.00). The authors were unable to supply more precise figures, so it was decided to assign a lower limit confidence interval so that the confidence intervals were symmetrical in logarithmic space around the point estimate. This enabled these studies to be entered into the analysis.

Heterogeneity was also assessed across the studies. To do this, 1/SE (a measure of precision) was plotted against In(hazard ratio per 10-unit SBP). The Spearman rank test was then used to calculate whether there was any relationship between these variables.

\section{RESULTS}

\section{Identification of reports}

The Medline and Embase searches for heart failure and prognosis returned 7132 citations, 10 of which met the inclusion criteria (table 1). This gave a total population of 8088 , with 29222 person-years of follow-up. The mean SBP across the included studies was $124.9 \mathrm{~mm} \mathrm{Hg}$.

\section{Relationship between SBP and mortality in CHF}

In all of the studies of CHF, a higher blood pressure was found to be associated with a better prognosis. The degree of benefit conferred by a $10 \mathrm{~mm} \mathrm{Hg}$ higher blood pressure varied between studies. We pooled the data to produce a consensus estimate and quantify its confidence intervals.

The pooled value for all studies included in the analysis gave a hazard ratio of 0.885 (95\% CI 0.87 to 0.90 ) for a $10 \mathrm{~mm} \mathrm{Hg}$ higher SBP in the population with CHF (fig 1). This equates to a decrease in mortality of $13.0 \%$ (95\% CI $10.6 \%$ to $15.4 \%$ ) for a $10 \mathrm{~mm} \mathrm{Hg}$ higher SBP. For a $1 \mathrm{~mm} \mathrm{Hg}$ higher SBP, the decrease in mortality is $1.2 \%$. In the lowest tertile of papers (mean SBP 109), a $10 \mathrm{~mm} \mathrm{Hg}$ higher SBP equated to an $18 \%$ decrease in mortality, with a $10 \%$ decrease in mortality per $100 \mathrm{~mm} \mathrm{Hg}$ higher SBP in the top tertile.

\section{Influence of ACE inhibitor and $\boldsymbol{\beta}$-blocker use}

Despite the difference in pharmacological treatments between studies, there was no significant difference in the relationship between SBP and mortality on subgroup analyses for patients receiving different doses of $\beta$ blockers $(p=0.93)$ or ACE inhibitors $(p=0.49)$ (fig 2$)$.

\section{Ischaemic versus non-ischaemic causes of heart failure}

The pooled value for mortality did not differ significantly when comparing predominantly ischaemic with predominantly nonischaemic aetiologies $(p=0.78)$. Division of studies dichotomously based on ejection fraction, however, did show a significantly different hazard ratio relating mortality and blood pressure ( $p=0.006$, fig 2$)$.

\section{Heterogeneity between studies}

The Spearman rank test for heterogeneity showed no significant evidence of heterogeneity between studies. On comparison of hazard ratio for a 10-unit change in SBP for studies divided into tertiles by mean SBP within the study showed a non-significant difference in hazard ratio for the highest tertile compared with the lowest tertile $(p=0.08)$ There was a non-significant association between mean SBP within a study and standardised hazard ratio (per 10-unit change in SBP) for that study $(p=0.26$, fig 3$)$.

\section{Comparison with non-heart failure studies}

Five relatively recent meta-analyses of the relationship between blood pressure and mortality in the general and/or hypertensive population were identified. ${ }^{23-8}$ This gave a total of over 12 million person-years of follow-up. The references for individual trial data were extracted. The large studies and meta-analyses of their results all showed a consistent relationship between SBP and mortality such that a higher SBP conferred a poorer prognosis in the non-heart failure population. ${ }^{9-11}$

\section{DISCUSSION}

In this study we have found that a higher SBP is consistently associated with a more favourable prognosis within the population with CHF. This is in contrast to the rest of the cardiac population, where a higher SBP is an adverse risk factor. This relationship was most marked in populations with a lower starting SBP.

\section{Physiological relationship between SBP and mortality in CHF}

A close relationship between SBP and mortality in CHF might be expected from consideration of cardiac physiology. The mean arterial blood pressure (MABP) is determined by the cardiac output (CO), the systemic vascular resistance (SVR) and the central venous pressure (CVP):

$\mathrm{MABP}=(\mathrm{CO} \times \mathrm{SVR})+\mathrm{CVP}$

In patients with cardiac disease but intact ventricular systolic function, the MABP is mostly determined by peripheral vasoconstriction. Therefore, if the systolic function is intact, a higher blood pressure indicates poorer elasticity of the arteries. 


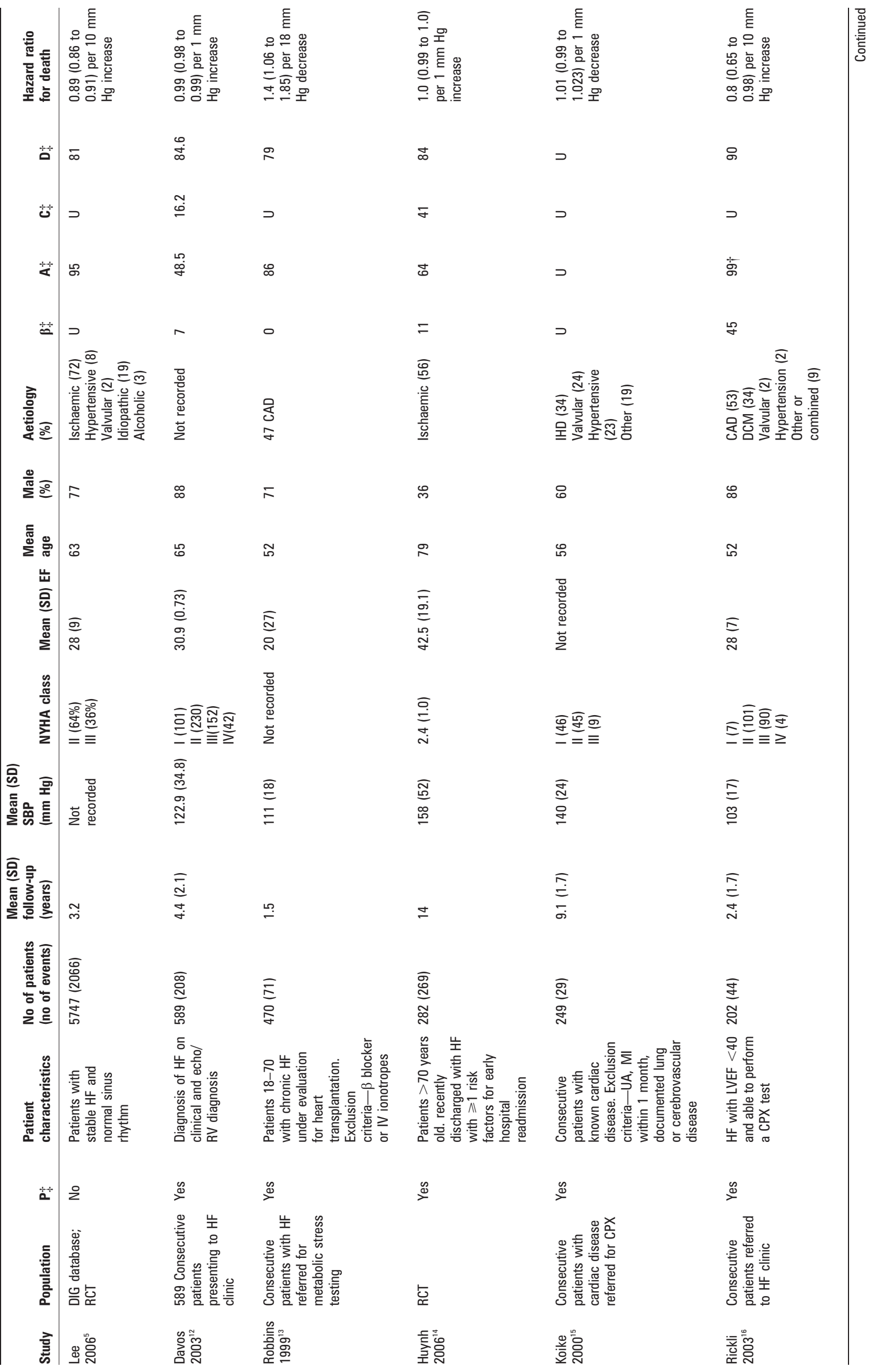




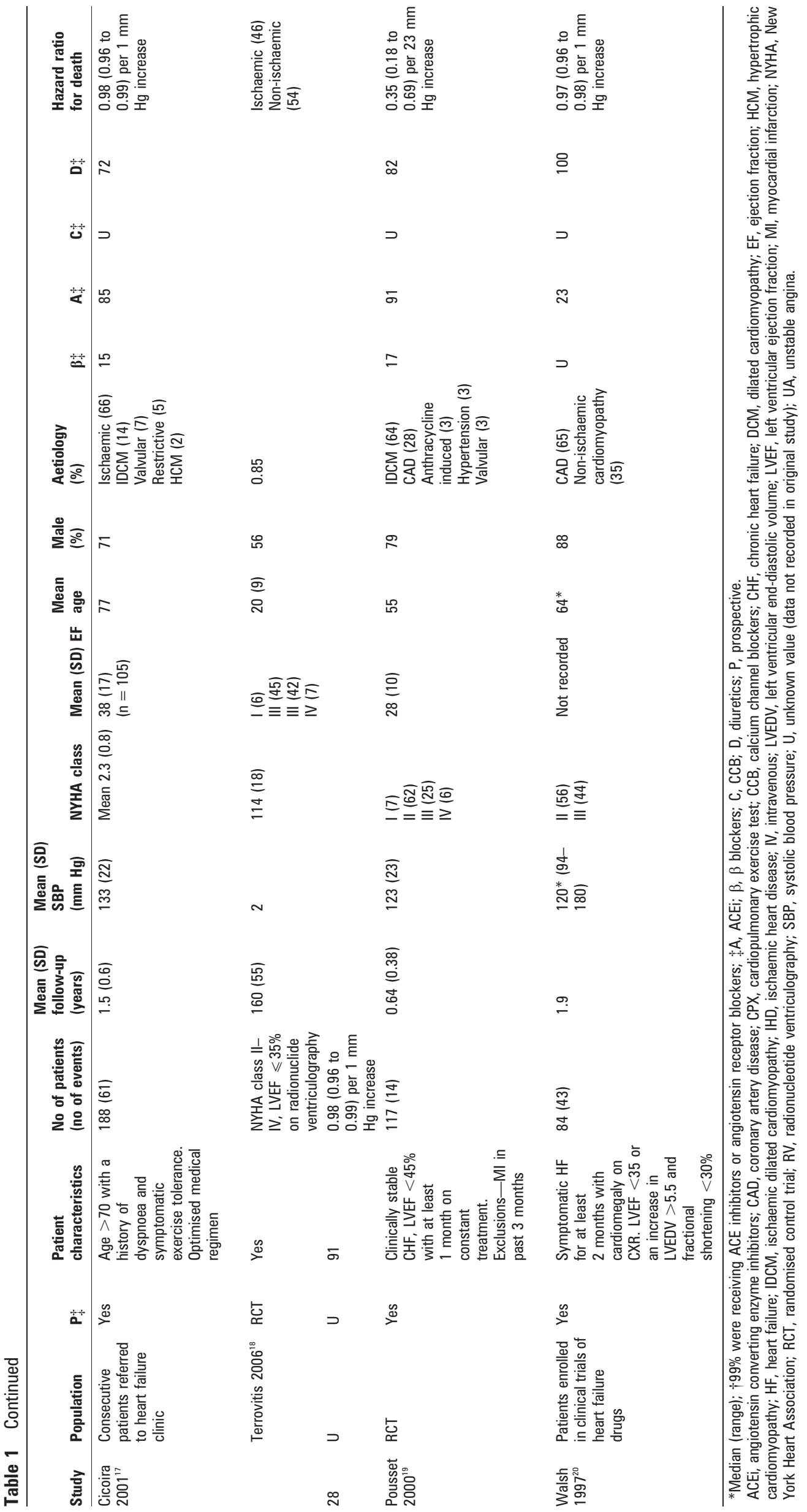




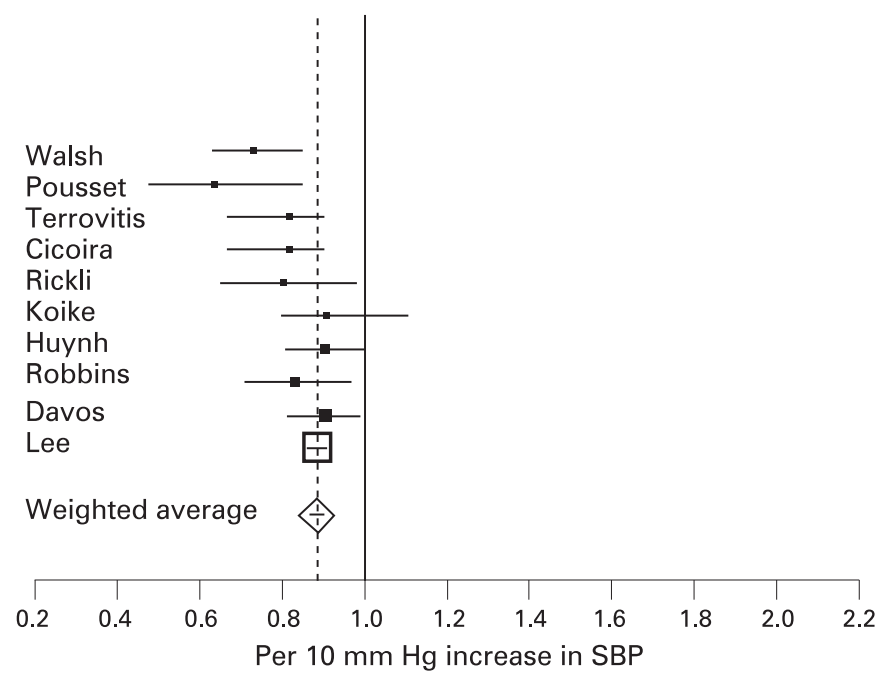

Figure 1 Hazard ratios for mortality associated with a $10 \mathrm{~mm} \mathrm{Hg}$ increase in systolic blood pressure (SBP). In the pooled heart failure studies, a $10 \mathrm{~mm} \mathrm{Hg}$ increase in SBP implies a $13 \%$ decrease in mortality. Conversely, in the non-heart failure population, an increase in SBP is associated with a poorer prognosis.

As shown in numerous meta-analyses, ${ }^{623}$ in patients without heart failure, a higher SBP is associated with a poorer prognosis.

The situation is entirely different within the population with CHF. In these patients, a low blood pressure reflects a low ejection fraction and cardiac output. The blood pressure is low because the heart is unable to pump any more vigorously to raise it. Therefore a higher blood pressure is associated with a decreased mortality as it serves as an indirect measure of cardiac function.

The MABP lies between the systolic and diastolic blood pressures. We chose to focus on SBP as there were no data on MABP in the studies analysed. Our study found a close and consistent relationship between SBP and mortality in CHF, with a higher SBP reflecting a better cardiac output and therefore more favourable prognosis.

\section{Bedside measures of prognosis in heart failure}

To link SBP to cardiac output, we should recognise that it is the difference between SBP and CVP that the heart is generating. Accordingly, poor cardiac function may result in low SBP or high CVP, or both. The prognostic value of high CVP has long been recognised clinically, and in recent years has been shown to have a close association with mortality. ${ }^{21}$ The chief attraction in blood pressure monitoring, however, is the relative reliability of measurement, even by people with relatively brief training.

\section{Quantification of the relationship between SBP and mortality}

The pooled value for all studies included in the analysis gave a decrease in mortality of $13.0 \%$ (95\% CI $10.6 \%$ to $15.4 \%$ ) per $10 \mathrm{~mm} \mathrm{Hg}$ higher SBP. The effect of a higher SBP is most marked in populations with a lower starting SBP, with an $18 \%$ lower mortality in the lowest tertile (mean SBP 109) and a 10\% decrease in the highest tertile (mean SBP 145) per $10 \mathrm{~mm} \mathrm{Hg}$ higher SBP. It is likely that the magnitude of effect associated with an increase in SBP continues to diminish such that a

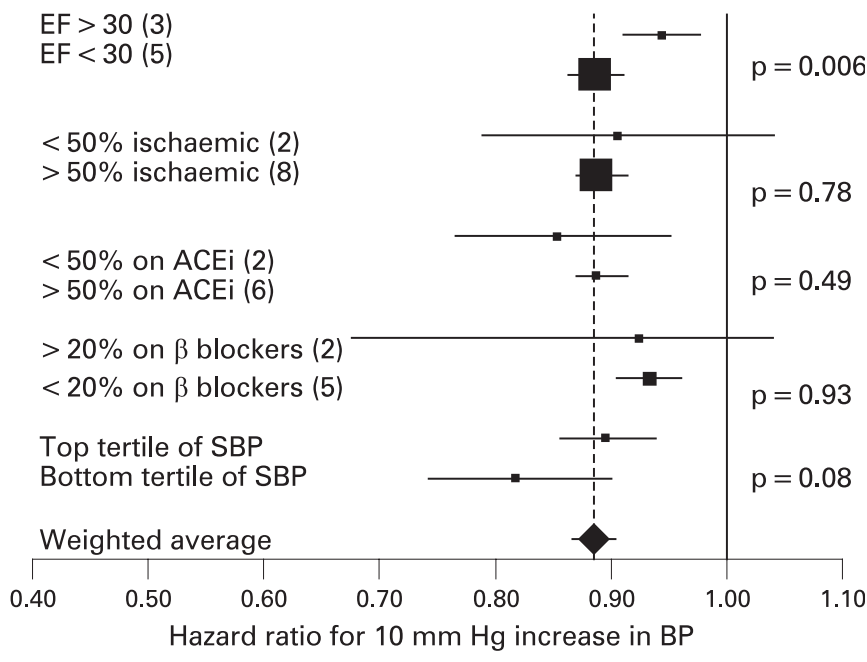

Figure 2 Subgroup analyses of included studies. EF, ejection fraction; SBP, systolic blood pressure.

$10 \mathrm{~mm} \mathrm{Hg}$ increase from 160 to $170 \mathrm{~mm} \mathrm{Hg}$ would confer a much smaller decrease in mortality, if any. Studies included in this analysis had a maximum SBP of $158\left(\mathrm{Huynh}^{14}\right)$, where there was a non-significant association between SBP and mortality. It may be that a level of blood pressure is reached beyond which a further increase confers an adverse prognosis, with a J-shaped relationship between SBP and mortality.

\section{Pharmacological effects on SBP}

There are many factors that influence both blood pressure and mortality in the population with CHF. Most drugs used in management of heart failure ( $\beta$ blockers, ACEi, diuretics) all serve to decrease blood pressure. In a patient with heart failure and low blood pressure, it is difficult to establish whether the blood pressure is low because of the advanced stage of the disease with poor cardiac output or whether it is low because the patient is being intensively managed pharmacologically.

The studies included in this analysis had a range of blood pressures and a heterogeneous sample of blood pressure lowering drugs. Despite this, there was a consistent relationship between SBP and mortality. On subgroup analysis, the only factor shown to affect this relationship significantly was ejection fraction. This was in keeping with previous studies on heart failure, which have shown that low blood pressure is an adverse prognostic sign in patients with impaired systolic

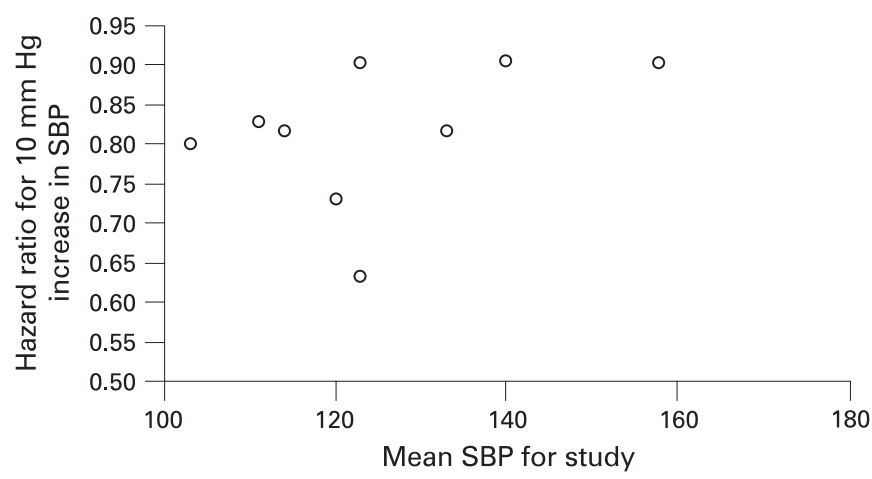

Figure 3 There was a non-significant relationship between standardised hazard ratio against the mean systolic blood pressure (SBP) for that population $(p=0.26)$. 
function but that it may in fact be a marker of good prognosis in those with intact systolic function. ${ }^{22}$

The lack of significant effect of pharmacological treatment on the relationship between blood pressure and mortality suggests a very powerful relationship between SBP and cardiac output, such that it overcomes confounding factors of medical treatment. This is also illustrated by the relatively consistent relationship between SBP and mortality despite a heterogeneous population with varying degrees of medical optimisation.

\section{Systolic and diastolic heart failure}

The studies included in this meta-analysis had a mean ejection fraction of 28.3 , indicating a population of predominantly systolic heart failure. The study by Huynh, however, has a mean ejection fraction of 42.5 , with only $36 \%$ men and an average SBP of $158 \mathrm{~mm} \mathrm{Hg}$. Additionally, 23\% of patients in the study by Koike had a hypertensive aetiology with a mean SBP of 140 and no EF reported. Hypertension is a common aetiology in diastolic heart failure, ${ }^{23}$ and these two studies may represent a population with predominantly diastolic heart failure. Interestingly, these are the only two studies identified where the relationship between SBP and mortality is not significant ( $\mathrm{HR}=0.99-1.0$ in Huynh and $\mathrm{HR}=0.99-1.023$ in Koike). Exclusion of these two studies does not alter the mean SBP per 10-unit change in SBP, as both studies have relatively small numbers.

\section{Implications for clinical practice}

While measurement of SBP is clearly not a substitute for measurements of left ventricular ejection fraction and other markers more closely associated with cardiac output, its attraction lies in its simplicity. SBP can be routinely measured at all outpatient visits and changes in SBP can be charted to monitor disease progression. While echocardiographic data may not exist before instigation of pharmacological treatment, SBP measurements would have been taken. The prognostic implications of changes in SBP over time have not been assessed in any study of which the authors are aware; however, this would be useful information to collect.

\section{Effect of antihypertensive drugs on SBP and cardiac output}

While we have shown a close relationship between blood pressure and mortality, it is obviously not our intention to suggest that antihypertensive drugs should be omitted in patients with heart failure. This study is entirely in keeping with the finding that many drugs that improve prognosis in systolic heart failure act to reduce blood pressure, including ACEi, angiotensin receptor blockers and $\beta$ blockers. The key reason is that across a population of patients with heart failure fully treated with these drugs the difference between their blood pressures is driven to some extent by differences in cardiac output.

The action of adding a prognostically beneficial vasodilator to a patient's treatment has the primary effect of reducing systemic vascular resistance, allowing the cardiac output to increase and the blood pressure to decrease. The cardiac output increase (which might be witnessed as an increase in ejection fraction) is beneficial, and the fall in blood pressure is a manifestation of the relief of intense vasoconstriction.

If our study were to be extrapolated to changes in blood pressure, it must be emphasised that it would only be possible to do this if there were no changes in vasodilator drugs. As long as the drug regimen were stable, a rise in blood pressure might be a good sign after implantation (or optimisation) of a cardiac resynchronisation therapy device.

\section{Potential relevance to haemodynamic optimisation of cardiac resynchronisation therapy}

This consistent finding across studies in heart failure lends indirect support to the concept of haemodynamic optimisation of cardiac resynchronisation therapy with non-invasive blood pressure monitoring. Rapid and reproducible algorithms for such optimisation are now available. ${ }^{24-26}$ These potentially extend the haemodynamic improvements already achieved from simply switching on the device. The landmark trials with cardiac resynchronisation therapy showed an increase in blood pressure, paralleling the improvements in symptoms and survival, for patients entered into the device arm. For example in the COMPANION trial, patients in the resynchronisation arm initially gained approximately $2 \mathrm{~mm} \mathrm{Hg} \mathrm{SBP}$ in comparison with the control arm, and went on to have a $18 \%$ relative reduction ( $95 \%$ confidence interval $1 \%$ increase to $42 \%$ reduction) in the combined end point of morbidity and mortality. ${ }^{27}$ Similarly the CARE-HF trial showed, at 3 months, that the increment in blood pressure attributable to being in the device arm was $5.8(95 \% \mathrm{CI} 3.5$ to 8.2$) \mathrm{mm} \mathrm{Hg}$, and the mortality reduction was $37 \%$ (95\% CI $23 \%$ to $49 \%$ ). ${ }^{28}$

In each case, the increase in SBP is likely to represent an improvement in cardiac output as a result of the resynchronisation. Since the SBP in patients with heart failure, unlike in the general population, is more closely governed by the cardiac output than peripheral resistance, this increase in SBP is likely to reflect an improved cardiac output and ejection fraction.

Whether increased blood pressure is a reasonable proxy for long-term outcomes has, however, been doubted, because of the general perception in cardiology that lower blood pressures are better. In this study, it becomes clear that increase in SBP, be it from resynchronisation itself or its optimisation, need not be assumed to be adverse, and might readily be beneficial. Of course, specific trials would be needed to determine this beyond doubt.

\section{Study limitations}

This type of study has many limitations. The populations chosen were heterogeneous, with a range of ejection fractions and mean systolic blood pressure, aetiologies and drugs, and were carried out at different time points. However, there was no significant association between hazard ratio and mean SBP for the studies included ( $p=0.16)$, which gives reassurance that the key findings are not being driven by a small subset of patients.

Additionally, with studies of different sample sizes there may be effects apparent in small studies that disappear with larger populations. However, there was no evidence of a relationship between size of study and hazard ratio $(p=0.09)$. For identification of studies to include in the analysis, there is also the problem of publication bias, where studies that did not find a significant relationship between mortality and blood pressure are less likely to publish their data. This was dealt with by using a search strategy that did not only return studies that looked at blood pressure and mortality, but found all studies that reported mortality data in a population with heart failure.

\section{CONCLUSION}

This study has shown that there is a close relationship between SBP and mortality in the population with CHF. This is contrary 
to the majority of cardiac disease, where a high SBP is an adverse prognostic sign. Quantitative awareness of this relationship between SBP and mortality may be of use in assessment or sequential follow-up of patients or where more extensive testing such as echocardiography is not available. It also gives indirect support to the concept of monitoring SBP as a simple haemodynamic marker during non-invasive haemodynamic optimisation of cardiac resynchronisation.

Acknowledgements: All authors contributed to the planning and analysis of the study, and interpretation of the results as well as to the writing of the paper. CER, ZIW and JED designed and performed the literature search and studies for inclusion. MF, EAF and CHM contributed to the statistical analysis of the results. JM and DPF designed and managed the overall study and planned the analysis methods. All authors approved the final manuscript. Guarantor-DPF.

Competing interests: None.

All researchers were independent of funders during the writing of this paper.

\section{REFERENCES}

1. Pfeffer MA, Swedberg K, Granger CB, et al. CHARM Investigators and Committees. Effects of candesartan on mortality and morbidity in patients with chronic heart failure: the CHARM-Overall programme. Lancet 2003;362:759-66.

2. Pocock SJ, McCormack V, Gueyffier F, et al. A score for predicting risk of death from cardiovascular disease in adults with raised blood pressure, based on individual patient data from randomised controlled trials. BMJ 2001;323:75-81.

3. Lewington S, Clarke R, Qizilbash N, et al. Prospective Studies Collaboration. Agespecific relevance of usual blood pressure to vascular mortality: a meta-analysis of individual data for one million adults in 61 prospective studies. Lancet 2002;360:1903-13.

4. Gheorghiade M, Abraham WT, Albert NM, et al. OPTIMIZE-HF Investigators and Coordinators. Systolic blood pressure at admission, clinical characteristics, and outcomes in patients hospitalized with acute heart failure. JAMA 2006;296:2217-26.

5. Lee TT, Chen J, Cohen DJ, et al. The association between blood pressure and mortality in patients with heart failure. Am Heart J 2006;151:76-83.

6. Staessen JA, Wang JG, Thijs L. Cardiovascular protection and blood pressure reduction: a meta-analysis. Lancet 2001;358:1305-15.

7. Turnbull F. Blood Pressure Lowering Treatment Trialists' Collaboration. Effects of different blood-pressure-lowering regimens on major cardiovascular events: results of prospectively-designed overviews of randomised trials. Lancet 2003;362:1527-35.

8. Staessen JA, Wang JG, Thijs L. Cardiovascular prevention and blood pressure reduction: a quantitative overview updated until 1 March 2003. J Hypertens 2003;6:1055-76.

9. Sesso HD, Stampfer MJ, Rosner B, et al. Two-year changes in blood pressure and subsequent risk of cardiovascular disease in men. Circulation 2000;102:307-12.

10. Stamler J, Neaton JD, Wentworth DN. Blood pressure (systolic and diastolic) and risk of fatal coronary heart disease. Hypertension 1989;13:12-12.
11. Tunstall-Pedoe H, Woodward M, Tavendale R, et al. Comparison of the prediction by 27 different factors of coronary heart disease and death in men and women of the Scottish Heart Health Study: cohort study. BMJ 1997;315:722-9.

12. Davos CH, Doehner W, Rauchhaus M, et al. Body mass and survival in patients with chronic heart failure without cachexia: the importance of obesity. J Card Fail 2003;9:29-35.

13. Robbins M, Francis G, Pashkow FJ, et al. Ventilatory and heart rate responses to exercise: better predictors of heart failure mortality than peak oxygen consumption. Circulation 1999;100:2411-7.

14. Huynh BC, Rovner A, Rich MW. Long-term survival in elderly patients hospitalized for heart failure: 14-year follow-up from a prospective randomized trial. Arch Intern Med 2006;166:1892-8.

15. Koike A, Koyama $Y$, Itoh $\mathrm{H}$, et al. Prognostic significance of cardiopulmonary exercise testing for 10-year survival in patients with mild to moderate heart failure. Jpn Circ J 2000;64:915-20.

16. Rickli H, Kiowski W, Brehm M, et al. Combining low-intensity and maximal exercise test results improves prognostic prediction in chronic heart failure. J Am Coll Cardiol 2003;42:116-22.

17. Cicoira M, Davos C, Florea V, et al. Chronic heart failure in the very elderly: clinical status, survival, and prognostic factors in 188 patients more than 70 years old. Am Heart J 2001:142:174-80.

18. Terrovitis JV, Anastasiou-Nana Ml, Alexopoulos GP, et al. Prevalence and prognostic significance of anemia in patients with congestive heart failure treated with standard vs high doses of enalapril. J Heart Lung Transplant 2006;25:333-8

19. Pousset F, Masson F, Chavirovskaia 0, et al. Plasma adrenomedullin, a new independent predictor of prognosis in patients with chronic heart failure. Eur Heart $J$ 2000;21:1009-14.

20. Walsh JT, Charlesworth A, Andrews R, et al. Relation of daily activity levels in patients with chronic heart failure to long-term prognosis. Am J Cardiol 1997; 79:1364-9.

21. Drazner MH, Rame JE, Stevenson LW, et al. Prognostic importance of elevated jugular venous pressure and a third heart sound in patients with heart failure. N Engl J Med 2001;345:574-81.

22. Ghali JK, Kadakia S, Bhatt A, et al. Survival of heart failure patients with preserved versus impaired systolic function: the prognostic implication of blood pressure. Am Heart J 1992;123:993-7.

23. Sanderson LE. Heart failure with a normal ejection fraction. Heart 2007:93:155-8

24. Whinnett ZI, Davies JER, Willson K, et al. Determination of optimal atrioventricular delay for cardiac resynchronization therapy using acute non-invasive blood pressure. Europace 2006;8:358-66.

25. Whinnett ZI, Davies JER, Nott G, et al. Efficiency, reproducibility and agreement of five different hemodynamic measures for optimization of cardiac resynchronization therapy. Int J Cardiol 2008;129:216-26.

26. Whinnett ZI, Davies JER, Willson K, et al. Hemodynamic effects of changes in AV and $\mathrm{V}$ delay in cardiac resynchronisation therapy show a consistent pattern: analysis of shape, magnitude and relative importance of AV and $\mathrm{W}$ delay. Heart 2006;92:1628-34.

27. Bristow MR, Saxon LA, Boehmer J, et al. Cardiac-resynchronization therapy with or without an implantable defibrillator in advanced chronic heart failure. N Engl J Med 2004;350:2140-50.

28. Cleland JG, Daubert JC, Erdmann E, et al. The effect of cardiac resynchronization on morbidity and mortality in heart failure. N Engl J Med 2005;352:1539-49. 